\title{
Retrospective Matched-cohort Analysis of Acute Pancreatitis Induced by 5-ASA-derived Drugs
}

SUPPLEMENTAL DIGITAL CONTENT

\begin{tabular}{|c|c|c|c|c|c|c|c|}
\hline \multicolumn{8}{|c|}{$\begin{array}{l}\text { SUPPLEMENTARY TABLE 1. Composition of the 5-ASA-DIAP and CAP Populations With Regard to Age, Sex, and } \\
\text { Etiology of AP }\end{array}$} \\
\hline \multirow[b]{2}{*}{ No. } & \multicolumn{4}{|c|}{ 5-ASA-DIAP Population } & \multicolumn{3}{|c|}{ CAP Population } \\
\hline & Age & Sex & Condition & Drug & Age & Sex & Etiology \\
\hline 1 & 12 & $\mathrm{M}$ & UC & Sulfasalazine & 12 & M & Ulcerative colitis \\
\hline 2 & 12 & M & $\mathrm{UC}$ & pH-dependent 5-ASA & 13 & M & Genetic \\
\hline 3 & 24 & $\mathrm{~F}$ & $\mathrm{UC}$ & 5-ASA & 24 & $\mathrm{~F}$ & Idiopathic \\
\hline 4 & 23 & $\mathrm{~F}$ & $\mathrm{UC}$ & pH-dependent 5-ASA & 23 & $\mathrm{~F}$ & Biliary \\
\hline 5 & 23 & $\mathrm{~F}$ & $\mathrm{UC}$ & Sulfasalazine & 23 & $\mathrm{~F}$ & Idiopathic \\
\hline 6 & 39 & $\mathrm{~F}$ & $\mathrm{UC}$ & Sulfasalazine & 39 & $\mathrm{~F}$ & Biliary \\
\hline 7 & 39 & $\mathrm{~F}$ & $\mathrm{UC}$ & Sulfasalazine & 39 & $\mathrm{~F}$ & Biliary \\
\hline 8 & 39 & $\mathrm{~F}$ & $\mathrm{UC}$ & Sulfasalazine & 39 & $\mathrm{~F}$ & ERCP \\
\hline 9 & 39 & $\mathrm{~F}$ & $\mathrm{UC}$ & Sulfasalazine & 39 & $\mathrm{~F}$ & Idiopathic \\
\hline 10 & 39 & $\mathrm{~F}$ & $\mathrm{UC}$ & Sulfasalazine & 39 & $\mathrm{~F}$ & Biliary \\
\hline 11 & 26 & M & $\mathrm{UC}$ & Time-dependent 5-ASA & 26 & M & Biliary \\
\hline 12 & 26 & M & $\mathrm{UC}$ & pH-dependent 5-ASA & 26 & M & Alcohol \\
\hline 13 & 29 & $\mathrm{~F}$ & $\mathrm{CD}$ & Sulfasalazine & 29 & $\mathrm{~F}$ & Biliary \\
\hline 14 & 41 & M & $\mathrm{CD}$ & 5-ASA + 5-ASA enema & 41 & M & Alcohol \\
\hline 15 & 29 & $\mathrm{~F}$ & $\mathrm{UC}$ & 5-ASA + 5-ASA enema & 29 & $\mathrm{~F}$ & Idiopathic \\
\hline 16 & 36 & $\mathrm{~F}$ & $\mathrm{UC}$ & 5-ASA & 36 & $\mathrm{~F}$ & Biliary \\
\hline 17 & 29 & $\mathrm{~F}$ & $\mathrm{CD}$ & 5-ASA & 29 & $\mathrm{~F}$ & ERCP \\
\hline 18 & 29 & $\mathrm{~F}$ & $\mathrm{CD}$ & Sulfasalazine & 29 & $\mathrm{~F}$ & Alcohol \\
\hline 19 & 34 & M & N/A & pH-dependent 5-ASA & 34 & M & Alcohol, hypertriglyceridemia \\
\hline 20 & 34 & M & N/A & pH-dependent 5-ASA & 34 & M & Idiopathic \\
\hline 21 & 31 & $\mathrm{~F}$ & $\mathrm{CD}$ & Time-dependent 5-ASA & 31 & $\mathrm{~F}$ & Biliary \\
\hline 22 & 35 & $\mathrm{~F}$ & $\mathrm{CD}$ & Time-dependent 5-ASA & 35 & $\mathrm{~F}$ & Biliary \\
\hline 23 & 25 & $\mathrm{~F}$ & $\mathrm{UC}$ & Time-dependent 5-ASA & 25 & $\mathrm{~F}$ & Idiopathic, genetic \\
\hline 24 & 37 & $\mathrm{~F}$ & $\mathrm{UC}$ & Sulfasalazine & 37 & $\mathrm{~F}$ & Diet \\
\hline 25 & 14 & $\mathrm{~F}$ & $\mathrm{CD}$ & Sulfasalazine & 14 & $\mathrm{~F}$ & Idiopathic \\
\hline 26 & 24 & $\mathrm{~F}$ & $\mathrm{UC}$ & 5-ASA & 24 & $\mathrm{~F}$ & Alcohol \\
\hline 27 & 24 & $\mathrm{~F}$ & $\mathrm{UC}$ & 5-ASA & 25 & $\mathrm{~F}$ & Biliary \\
\hline 28 & 34 & M & $\mathrm{UC}$ & 5-ASA & 34 & M & Alcohol \\
\hline 29 & 34 & M & $\mathrm{UC}$ & 5-ASA & 34 & M & Alcohol \\
\hline 30 & 30 & M & $\mathrm{UC}$ & 5-ASA & 30 & M & Idiopathic \\
\hline 31 & 19 & M & $\mathrm{CD}$ & 5-ASA & 18 & M & Alcohol \\
\hline 32 & 12 & M & $\mathrm{UC}$ & Olsalazine + 5-ASA enema & 13 & M & Idiopathic \\
\hline 33 & 13 & $\mathrm{~F}$ & $\mathrm{UC}$ & Sulfasalazine & 13 & $\mathrm{~F}$ & Biliary \\
\hline 34 & 12 & $\mathrm{~F}$ & $\mathrm{UC}$ & Sulfasalazine & 12 & $\mathrm{~F}$ & Biliary \\
\hline 35 & 12 & $\mathrm{~F}$ & $\mathrm{UC}$ & Olsalazine & 12 & $\mathrm{~F}$ & Biliary \\
\hline 36 & 40 & M & $\mathrm{UC}$ & 5-ASA & 40 & M & Anatomical \\
\hline 37 & 63 & $\mathrm{~F}$ & $\mathrm{UC}$ & pH-dependent 5-ASA & 63 & $\mathrm{~F}$ & Alcohol \\
\hline 38 & 31 & $\mathrm{~F}$ & $\mathrm{CD}$ & time-dependent 5-ASA & 31 & $\mathrm{~F}$ & Biliary \\
\hline 39 & 31 & $\mathrm{~F}$ & $\mathrm{CD}$ & time-dependent 5-ASA & 30 & $\mathrm{~F}$ & ERCP \\
\hline
\end{tabular}




\begin{tabular}{lccccccc}
\hline \multicolumn{7}{l}{ TABLE 1. (Continued) } & \multicolumn{7}{l}{} \\
\hline 40 & 28 & $\mathrm{~F}$ & $\mathrm{UC}$ & sulfasalazine & 28 & $\mathrm{~F}$ & Biliary, anatomical \\
41 & 33 & $\mathrm{M}$ & $\mathrm{UC}$ & 5-ASA & 33 & $\mathrm{M}$ & Alcohol \\
42 & 7 & $\mathrm{~F}$ & $\mathrm{UC}$ & time-dependent 5-ASA & 6 & $\mathrm{~F}$ & Idiopathic \\
43 & 7 & $\mathrm{~F}$ & $\mathrm{UC}$ & time-dependent 5-ASA & 6 & $\mathrm{~F}$ & Idiopathic \\
44 & 10 & $\mathrm{M}$ & $\mathrm{UC}$ & pH-dependent 5-ASA & 10 & $\mathrm{M}$ & Idiopathic \\
45 & 12 & $\mathrm{M}$ & $\mathrm{CD}$ & 5-ASA enema & 12 & $\mathrm{M}$ & Anatomical \\
46 & 37 & $\mathrm{M}$ & $\mathrm{UC}$ & sulfasalazine & 37 & $\mathrm{M}$ & Alcohol \\
47 & 32 & $\mathrm{~F}$ & $\mathrm{CD}$ & pH-dependent 5-ASA & 32 & $\mathrm{~F}$ & Biliary \\
48 & 32 & $\mathrm{~F}$ & $\mathrm{CD}$ & pH-dependent 5-ASA & 32 & $\mathrm{~F}$ & Biliary \\
49 & 19 & $\mathrm{M}$ & $\mathrm{UC}$ & 5-ASA & 20 & $\mathrm{M}$ & Diet \\
50 & 18 & $\mathrm{M}$ & $\mathrm{UC}$ & 5-ASA + 5-ASA enema & 17 & $\mathrm{M}$ & Idiopathic \\
51 & 35 & $\mathrm{M}$ & $\mathrm{UC}$ & pH-dependent 5-ASA & 35 & $\mathrm{M}$ & Hypertriglyceridemia \\
\hline \multicolumn{7}{l}{ UC indicates ulcerative colitis; CD, Crohn's disease; N/A, not applicable (condition not specified); ERCP, endoscopic } \\
\multicolumn{7}{l}{ retrograde cholangiopancreatography. }
\end{tabular}

